\title{
Kinematic Approach to the 24th Solar Cycle Prediction
}

\author{
Vladimir Kaftan \\ Geophysical Center of the Russian Academy of Sciences, Molodezhnaya Street 3, 119296 Moscow, Russia \\ Correspondence should be addressed to Vladimir Kaftan, v.kaftan@gcras.ru
}

Received 14 November 2011; Revised 20 January 2012; Accepted 10 February 2012

Academic Editor: J. Javaraiah

Copyright ( $) 2012$ Vladimir Kaftan. This is an open access article distributed under the Creative Commons Attribution License, which permits unrestricted use, distribution, and reproduction in any medium, provided the original work is properly cited.

\begin{abstract}
The results of kinematic modeling of the 24th solar cycle (SC) are discussed. Time series of solar and cosmic ray monthly mean indices were received from web resources of international data centers. The previous prediction of the solar cycle shape using kinematic modeling technique demonstrated rather close agreement with the final phase of the SC23 and relatively large magnitude of SC24. The forecast of 2005 is updated with respect to the modern observation of monthly mean data. The study allows concluding that the SC24 magnitude will probably near the previous cycle. Predicted moments of the maximum monthly mean values are expected in July-September 2012. The uncertainty of this interval is about $\pm 1-2$ months. The maximum monthly mean estimation can reach $130 \pm 20$ relative sunspot number units. The mean amplitude of the generalized cycle shape is expected to be close to the 23rd maximum mean height. The SC24 form can be relatively narrow, and the cycle will probably be shorter than 10 years.
\end{abstract}

\section{Introduction}

As it was concluded by the special solar cycle prediction panel, the sample of different forecasts was separated in two main predictions of reasonably large or small SC24 magnitudes at 2007 (http://www.swpc.noaa.gov/SolarCycle/SC24/ index.html). The first consensus proposed two rather different amplitudes of 140 and 90 sunspot number units as the most probable estimations, but later it was reduced to the lowest variant of 90. Another information on different SC prediction comparison can be seen at the Jan Janssens web resource http://users.telenet.be/j.janssens/SC24.html/. Some additional high magnitude forecasts can be found in [1-5]. Another low prediction is published in [6]. Comparison analyses of different approaches to SC24 prediction are presented in papers $[7,8]$.

All the predictions were classified according to the used technique as physical, statistical, and mixed. It is remarkable that the physical predictions have lower magnitudes than statistical and mixed ones, with the exception of Dikpati researches [1-5]. The most of the used forecasting techniques are statistical.

One of the statistical techniques called as kinematical approach previously allowed to predict the long final stage of the 23rd cycle and high amplitude of the 24th one (http:// journals.cambridge.org/action/displayAbstract?fromPage= online\&amp;aid=288312). As a matter of fact, the final stage of SC was predicted accurately using this technique in a contrast of many other predictions. The experience of the kinematic technique usage made the author think that it could provide more or less precise predictions only for the $\sim 5$ year forecasting interval in the case of about 50 year length monthly mean data processing. The last prediction of the current solar cycle shape was performed in 2005 and published in 2006. It is demonstrated relatively large monthly mean sunspot index closed to 160 sunspot number units [9]. Nowadays, this prediction is tested and updated taking into account the up to date monthly data.

\section{Observation Data and Modeling Technique}

The time series of the monthly mean values of solar indices were used in the analyses. The international sunspot numbers (Ri) were received from the website (http://sidc.oma .be/index.php3). The data of the $10.7 \mathrm{~cm}$ solar radio flux were obtained from the website (http://www.spaceweather.ca/ sx-eng.php). The time series of monthly mean sunspot areas $(\mathrm{S})$ was taken from the Internet site (http://solarscience.msfc.nasa.gov/greenwch/sunspot_area.txt). The cosmic 
ray time series of observation with monthly intervals from the Moscow observation station was used in the research as additional indirect data on solar variability (ftp:// cr0.izmiran.rssi.ru/COSRAY!/FTP_DDMM/Data/). The time span of every series was varied between different analyses as described below.

The observation data were processed using the numerical technique called as the sequential analysis of dominated harmonics method. The method was used firstly for the Caspian Sea level change prediction [10] but fully described and published in the thesis [11]. It is briefly described as follows.

The results of repeated observation $y$ of irregular time series are expressed in terms of $k$ unknown parameters $x$ and a temporal argument $t$ as

$$
y_{i}=f\left(x_{1}, x_{2}, \ldots, x_{k}, t\right) .
$$

This function has to be consisted of the number of periodical ( sinusoidal) components which can be superposed to unidirectional trend changes. Thereafter, the connection equation can be written as

$$
y_{t}=b_{0}+b_{1} t+\sum_{j=1}^{n}\left(M_{j}+A_{j} \sin \left(\omega_{j} t+\phi_{j}\right)\right),
$$

where $b_{0}$ is the value of the linear trend function at the initial moment, $b_{1}$ is the linear coefficient of the first order (linear velocity), $M_{j}$ is the ordinate of an oscillation axis of the $j$ th harmonic, and $A_{j}, \omega_{j}$, and $\varphi_{j}$ denote the amplitude, the angular frequency, and angular phase of the $j$ th harmonic, respectively.

In the absence of a priory information about a number and a contribution level of periodical harmonics in the whole character of variations, the iteration step by step procedure seems to be efficient in the analysis.

At the first stage, it is natural to determine and remove a trend component from the series using the equation

$$
y=b_{0}+b_{1} t
$$

Correspondent correction equation system will be presented as

$$
v_{l}=\left[\begin{array}{cc}
1 & t_{1} \\
1 & t_{2} \\
\cdots & \cdots \\
1 & t_{n}
\end{array}\right] \cdot\left[\begin{array}{ll}
b_{0} & b_{1}
\end{array}\right]-\left[\begin{array}{c}
-y_{1} \\
-y_{2} \\
\cdots \\
-y_{n}
\end{array}\right]=A_{l} x_{l}+l_{l},
$$

where $v_{l}$ denotes a residual random noise term, $l$ index reflects a linear part of variation, and $n$ is the number of values in the analyzed time series.

This task can be solved under condition of

$$
v_{l}^{T} P v_{l}=\min ,
$$

where $P$ is an $n \times n$ weight matrix.

For the common case, we can have a priory inverse weight matrix

$$
Q_{l}=P^{-1}=\left[\begin{array}{cccc}
q_{11} & q_{12} & \cdots & q_{1 n} \\
q_{21} & q_{22} & \cdots & q_{2 n} \\
\cdots & \cdots & \cdots & \\
q_{n 1} & q_{n 2} & \cdots & q_{n n}
\end{array}\right]
$$

Then, the least squares solution of (4) will be given as

$$
x_{l}=-\left(A_{l}^{T} P A_{l}\right)^{-1} A_{l}^{T} P l_{l}
$$

At the second step, removing the modeled linear component from the initial time-series, subtracting (3) from (2), we will search for dominating harmonic components.

The model for the residual time series will be presented as

$$
y=\sum_{j=1}^{k}\left(M_{j}+A_{j} \sin \left(\omega_{j} t+\phi_{j}\right)\right)
$$

Its unknown parameters will be searching consequently for every harmonic component

$$
y=M_{j}+A_{j} \sin \left(\omega_{j} t+\phi_{j}\right) .
$$

Assigning by rough model parameters

$$
x_{0}=\left[M_{0}, A_{0}, \omega_{0}, \varphi_{0}\right]^{T}
$$

of the harmonic (9), after the linearization by first terms of Taylor series, we will receive a correction equation matrix $B$ :

$$
v=B_{x}+l
$$

and represent it as

$B_{0}=\left[\begin{array}{cccc}1 & \sin \left(\omega_{o} t_{1}+\phi_{0}\right) & A_{o} t_{1} \cos \left(\omega_{0} t_{1}+\phi_{0}\right) & A_{o} \cos \left(\omega_{0} t_{1}+\phi_{0}\right) \\ 1 & \sin \left(\omega_{o} t_{2}+\phi_{0}\right) & A_{o} t_{2} \cos \left(\omega_{0} t_{2}+\phi_{0}\right) & A_{o} \cos \left(\omega_{0} t_{2}+\phi_{0}\right) \\ \cdots & \cdots & \cdots & \cdots \\ 1 & \sin \left(\omega_{o} t_{n}+\phi_{0}\right) & A_{o} t_{n} \cos \left(\omega_{0} t_{n}+\phi_{0}\right) & A_{o} \cos \left(\omega_{0} t_{n}+\phi_{0}\right)\end{array}\right]$.

Free terms of correction equations will be received as

$$
l_{0}=\left[\begin{array}{c}
y_{1}-\left(M_{0}+A_{0} \sin \left(\omega_{0} t_{1}+\phi_{0}\right)\right) \\
y_{2}-\left(M_{0}+A_{0} \sin \left(\omega_{0} t_{2}+\phi_{0}\right)\right) \\
\cdots \\
y_{i}-\left(M_{0}+A_{0} \sin \left(\omega_{0} t_{i}+\phi_{0}\right)\right)
\end{array}\right] .
$$

As the initial model (9) is nonlinear, we will search for the unknown parameters $x$ numerically by the iteration procedure.

The iteration process will be written as

$$
x_{r+1}=x_{r}+f\left(x_{r}\right), \quad(r=0,1,2, \ldots),
$$

where $r$ is an iteration number.

Estimation of the vector-function $f\left(x_{r}\right)$ will be given by

$$
f\left(x_{r}\right)=-\left(B_{r}^{T} P B_{r}\right)^{-1} B_{r}^{T} P l_{r} .
$$

Then, the iteration process (14) will take the form

$$
x_{r+1}=x_{r}-\left(B_{r}^{T} P B_{r}\right)^{-1} B_{r}^{T} P l_{r} .
$$

Signing by some neglecting difference $\Delta_{x}$, we continue iterations for the moment when $\left[x_{r+1}-x_{r}\right]$ will be less than or equal to $\Delta_{x}$. 
Hence, the results of the last iteration will be accepted as final estimations of the model (9) parameters

$$
x=\left[\begin{array}{llll}
M & A & \omega & \phi
\end{array}\right]^{T} .
$$

In order to estimate a statistical confidence of the resulted characteristics, we use the estimation of the unit weight standard

$$
\mu=\sqrt{\frac{v^{T} P v}{n-4}},
$$

where $v$ is an $n \times 1$ vector of residuals (11) computed with the use of (17).

Empirical standards $S$ of harmonic parameter estimations will be determined as

$$
\left[\begin{array}{l}
s_{M} \\
s_{A} \\
s_{\omega} \\
s_{\phi}
\end{array}\right]=\mu \sqrt{Q_{i i}}=\mu \sqrt{\left(B_{r}^{T} P B_{r}\right)_{i i}{ }^{-1}},
$$

where $Q_{i i}=\left(B_{r}^{T} P B_{r}\right)_{i i}^{-1}$ are diagonal elements of the inversed weight matrix of parameters.

Then, we will compute normalized parameter estimations

$$
Z=\left[\begin{array}{c}
M / s_{M} \\
A / s_{A} \\
\omega / s_{\omega} \\
\phi / s_{\phi}
\end{array}\right] .
$$

Assigning a confidence limit $t \geq Z_{q \%}$, we determine a confidence level of received estimations. Depending on a solving task, a confidence limit $t$ is assigned using a normal distribution. In most of research, we can consider solved parameters as statistically confident when $Z \geq 2$.

Having a reason to consider a harmonic to be statistically confident, we receive an approximation function (9) which has to be subtracted from the previous residual time series. We follow the stepwise process of dominate harmonic determination (11)-(20) before we will be sure of the absence of confident components in the residual time series. The criterion of finishing an iteration procedure is a level of a closeness of unit weight standards of last harmonics.

The technique considered in this work is used in a statistical modeling of time variations in observed characteristics. This method can be considered among the periodogram analysis methods the general theory of which was described, for example, in [12]. In a contrast to the majority of the known methods, the technique used in the present work does not require regularity of observations. The specific feature of this technique consists also in an organized order of determining dominating sinusoidal components with decreasing amplitudes. The technique appears to be possible to individually estimate the accuracy of determination of required dominating harmonics.

\section{Former Results of the 24th Solar Cycle Prediction}

The above-described method was used in the modeling and prediction of the $23 \mathrm{rd}$ solar cycle form using Ri and $10.7 \mathrm{~cm}$

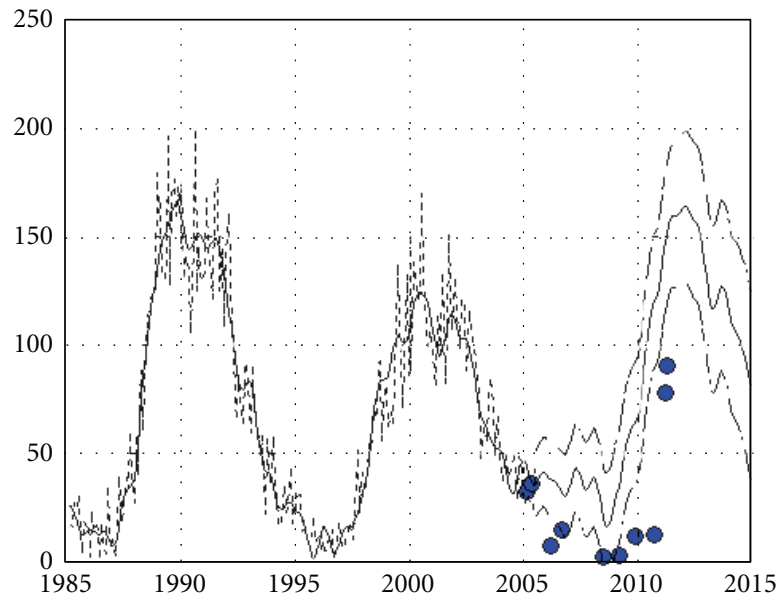

Figure 1: Model, prediction, and epignosis of the monthly mean Ri pattern. Dotted line demonstrates the real data. Dashes show the one sigma intervals. Blue dots are principal epignosis control marks. Abscissa axis fix years of the last modeled and predicted intervals. Ordinate axis shows international sunspot number values.

radio flux data [13]. The prediction was appeared to be rather close to the real SC23 outline. Predicted monthly mean maximum was coincide with the official maximal smoothed value but monthly indices themselves had relative standard error of $10-15 \%$. Moreover, several retrospective SC predictions using the technique showed rather accurate association with reality at least for the 5-year predicting intervals after the 50-year observation of the monthly mean indices.

The practice proved that the best predicting results were received in a case of relatively short solar time series usage. Predictions usually became confidently lower than reality in the use of hundreds years long-time series of monthly mean indices. This feature can be explained by the less accurate ancient observation and a possible miss of some cycles as it was discussed in publications.

It is necessary to remark that a quantity of a sample of determined periodically components is normally about several tenses for a 50-year monthly mean solar time series. In these cases, a statistical degree of freedom is more than 400 , that demonstrates a level of an effectiveness of the used approximation method. The time spans of series of relative sunspot numbers and sunspot areas were spread from 1950.0. Used series of $10.7 \mathrm{~cm}$ solar radio flux and cosmic ray indices had begun from 1947.0 and 1958, respectively. The final moments of all-time series were limited by the beginning date for the last and the current predictions.

The last SC predictions using the sequential analysis of dominated harmonics were made in 2004-2005 (see Figures 1-3) [9]. Three types of the solar indices were processed, and the 24th SC pattern was forecasted using about 50-year time series of the monthly mean values. Today, it is possible to check the accuracy and reliability of these forecasts. The original curves of the predictions and control marks are showed Figures 1-3. Blue marks are added on the published figures to show the forecast effectiveness. 


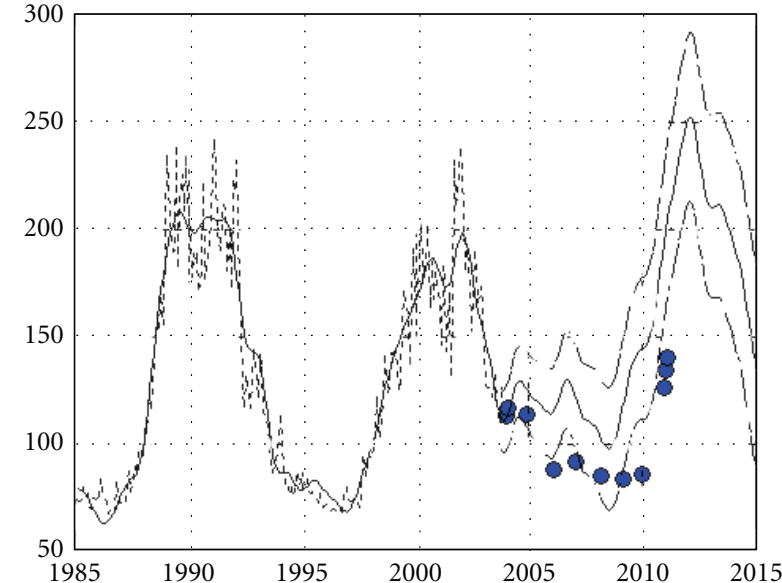

Figure 2: Model, prediction, and epignosis of the monthly mean indices of $10.7 \mathrm{~cm}$ radio flux (S.F.U) pattern. Dotted line demonstrates the real data. Dashes show the one sigma intervals. Blue dots are principal epignosis control marks. Abscissa axis fix years of the last modeled and predicted intervals. Ordinate axis shows S.F.U. values.

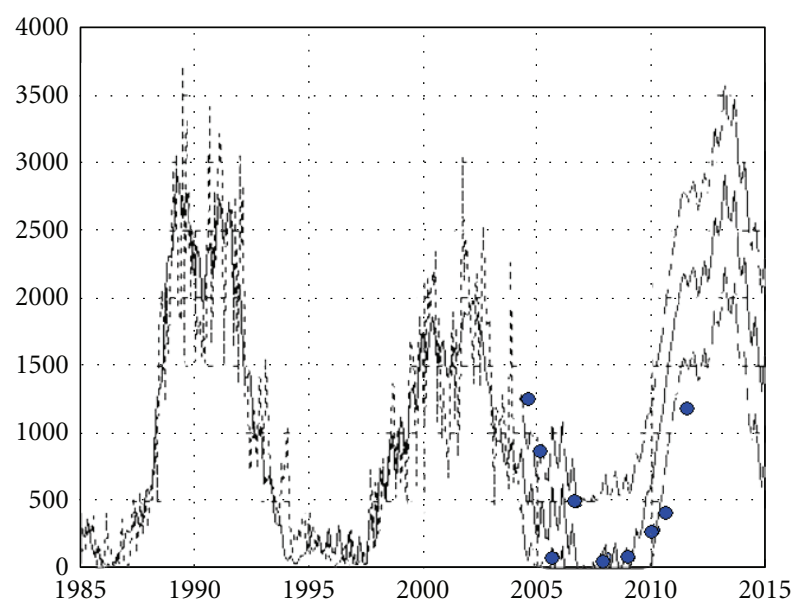

Figure 3: Model, prediction, and epignosis of the monthly mean sunspot areas pattern. Dotted line demonstrates the real data. Dashes show the one sigma intervals. Blue dots are principal epignosis control marks. Abscissa axis fix years of the last modeled and predicted intervals. Ordinate axis shows sunspot area values in units of millionths of a hemisphere.

It is necessary to explain that the one sigma tendencies were previously determined like mean square values of true errors in five retrospective forecasts of the way of behavior of $\mathrm{Ri}$ indices.

The analysis of the correlation between the different pair of indices was allowed to determine the correspondent linear regression formulas which were used in one sigma interval determination for the S.F.U and sunspot area values.

The tests of the previous SC forecasts demonstrate that the best prediction is present in the 5-year interval as it was expected from the earliest experiments. One can see that the true errors of the forecast are near to one sigma margins.
Actual solar indices are systematically lower than the forecasted positions for the Ri and 10.7 radio flux variations (see Figures 1 and 2). The character of the discrepancies is slightly different for the sunspot areas (see Figure 3). All predictions demonstrate the visible phase shift between the predicted and real data after the 5-year prognostic interval.

The general conclusions of the former SC behavior forecast were the next $[9,14]$.

(1) The minimal monthly mean indices were expected for the 2006.5-2008.

(2) The final stage of the 23rd solar cycle was expected to be reasonable long and have some local maxima.

(3) The 24th solar cycle monthly mean index was expected to be near $150 \pm 35$ Ri.

The final phase was estimated especially for the main solar indices together with galactic cosmic ray data [15]. We have to remark that the general features of the SC23 final stage were predicted rather accurately. Especially the about 12.6-year abnormal length of the 23rd cycle [16] is closely coincided with prediction described.

In a reference to the intercycle minimum phase, we have to give a passing mention to some principal studies. The nature of the minimal phase between 23rd and 24th solar cycles was carefully analyzed using $10.7 \mathrm{~cm}$ solar radio flux indices in the paper [17]. The interval between contiguous main solar cycle maxima is thoroughly studied in [18].

Today, it is a propitious moment for updating the published previous prediction because the amplitude and the time of maximum activity are not obvious yet.

\section{24th Solar Cycle Prediction Update}

The same data type sets updated by the modern observed values were used for the correction of the former SC24 prediction. Analyzed time series were consisted of monthly mean indices. Data archive addresses are denoted above. The main task for the moment is improving the estimations of the amplitude and the length of the SC24.

New data processing using the sequential analysis of dominated harmonics method was performed for the sunspot numbers, $10.7 \mathrm{~cm}$ radio flux indices, and sunspot area time series. Cosmic ray observations from the Moscow station were used too. The results of new SC24 predictions are illustrated in Figures 4, 7, 5, and 6.

As we can see from the Figures 4-6, new modeling of the four difference solar and cosmic processes demonstrates their probable future behavior closed each other. The amplitude of the SC24 is expected to be near to the previous cycle mean height. The International Sunspot Number prediction curve shows even larger modeled magnitude $130 \pm 20 \mathrm{Ri}$. The maximal phase interval can probably begin from the middle to the end of 2012.

The interesting fact is that the previous high magnitude SC24 forecast of the SC prediction panel coincides with the presented prediction in general in a reference to the magnitude and relatively narrow shape. It can be seen in comparison with Figure 8 taken from the original 


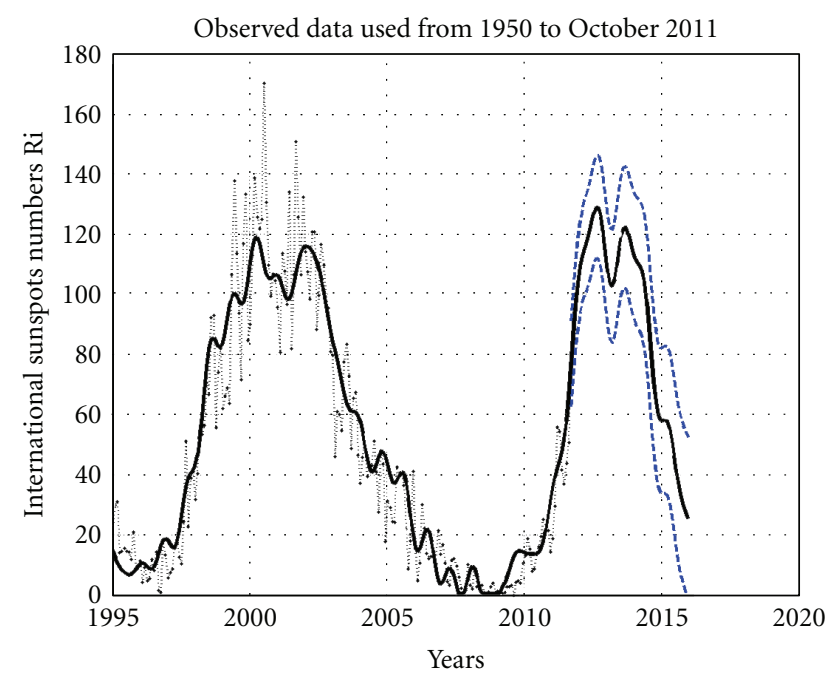

FIGURE 4: SC24 shape prediction for monthly mean sunspot number variation. Dotted line demonstrates the real data. Dashes show the one sigma intervals. Solid is the modeled curve.

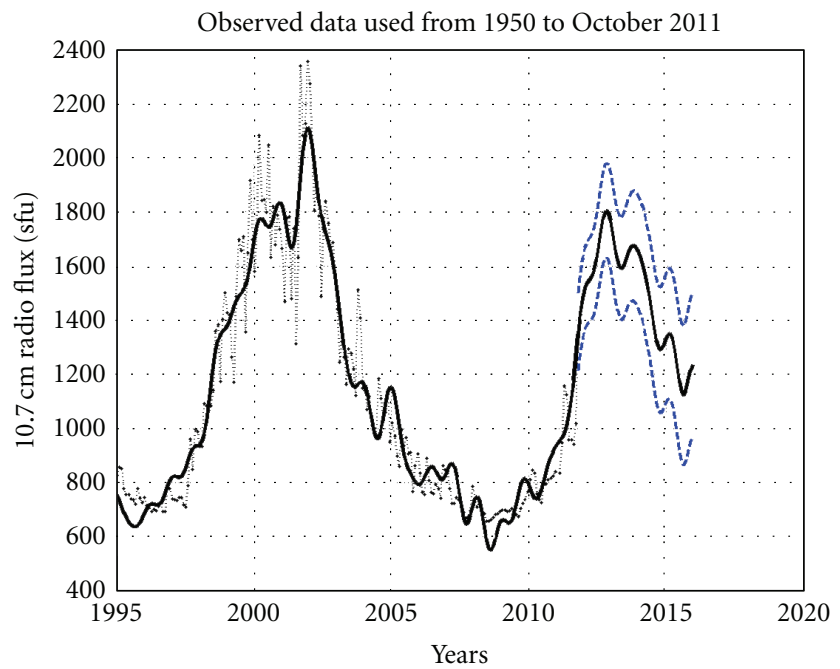

FIGURE 5: SC24 shape prediction of monthly mean indices for $10.7 \mathrm{~cm}$ radio flux variation. Dotted line demonstrates the real data. Dashes show the one sigma intervals. Solid is the modeled curve.

web resource (http://www.swpc.noaa.gov/SolarCycle/SC24/ index.html). But later, the panel researchers changed their opinion to the lowest variant which is better coinciding with the presented predictions in relation to the time of a maximum from the middle of 2012 to the beginning of 2013 (Figure 9).

\section{Conclusions}

Three types of solar data were used in the kinematic modeling for the previous prediction [9] updating. All new predictions demonstrate that 24th cycle maximum stage will probably occur at the middle of 2012 or a bit later. Predicted moments of the maximum monthly mean values are fallen

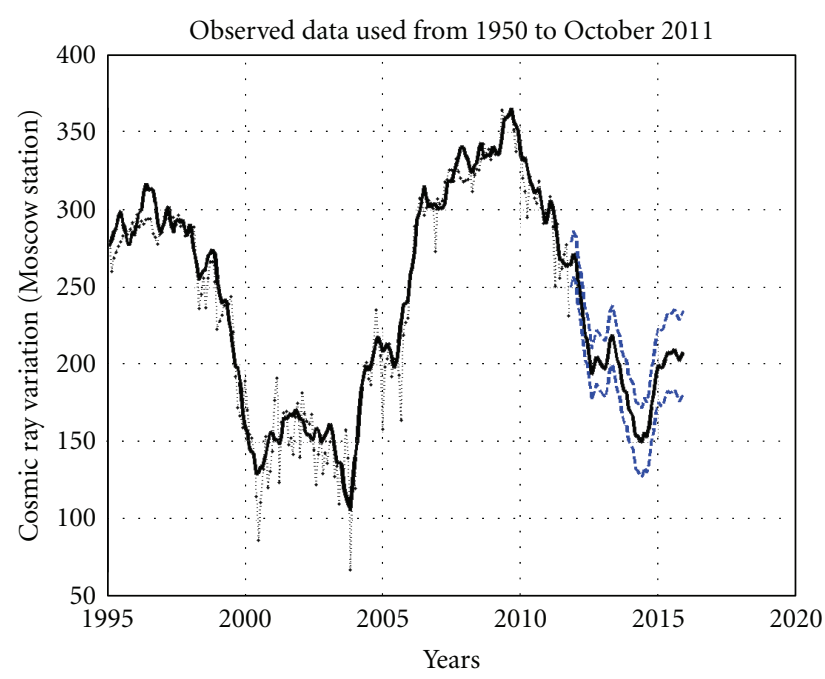

FIGURE 6: SC24 shape prediction for cosmic ray monthly index variation. Dotted line demonstrates the real data. Dashes show the one sigma intervals. Solid is the modeled curve.

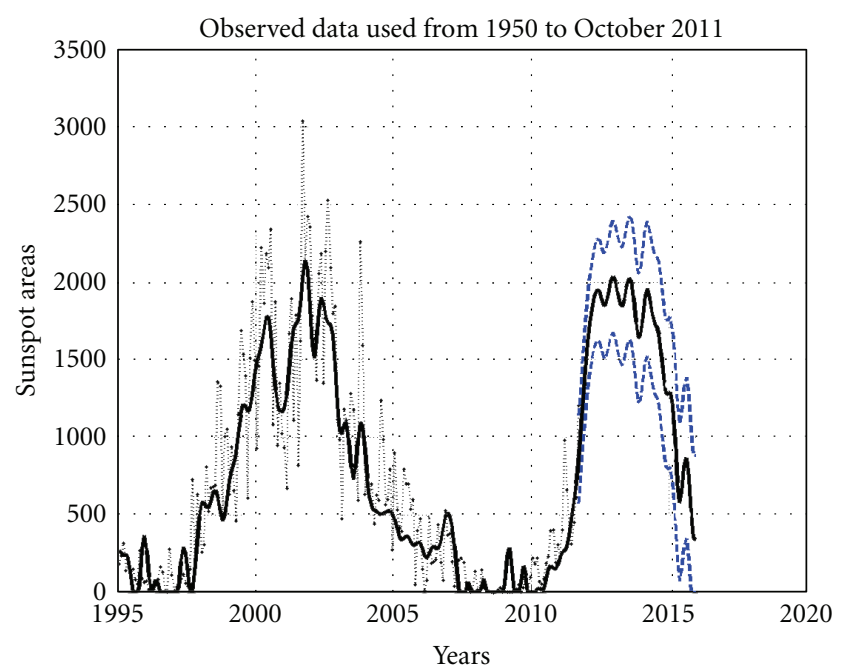

FIGURE 7: SC24 shape prediction for monthly mean sunspot areas variation. Dotted line demonstrates the real data. Dashes show the one sigma intervals. Solid is the modeled curve.

into July-September 2012. The uncertainty of this interval is about $\pm 1-2$ months. The mean amplitude of the general cycle shape is expected to be close to the 23rd maximum as it is seen from Figures 7, 5, and 6. The SC24 will continue not long. It will be probably shorter than 10 years. The beginning of the next minimum stage is preliminary predicted in 2017. The maximal monthly mean value of sunspot number $\mathrm{Ri}$ is forecasted as $130 \pm 20$. More than $2000 \pm 350$ units of millions of hemisphere are predicted for the maximal monthly mean sunspot area index. The $10.7 \mathrm{~cm}$ radio flux maximal monthly value is expected at the level of $200 \pm 20$ sfu. Cosmic ray variation at the Moscow station is modeled too as additional source of data. Its reversed magnitude is processed to be slightly lower than the level of the last solar cycle shape. 


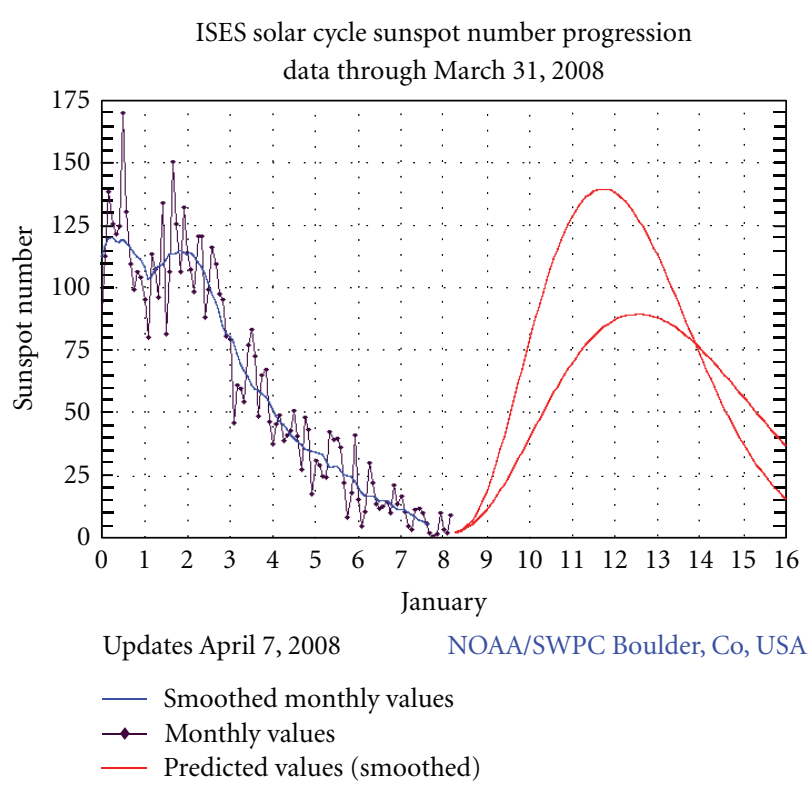

Figure 8: The first international consensus in SC24 prediction dated April 7, 2008 (http://www.swpc.noaa.gov/SolarCycle/SC24/index.html).

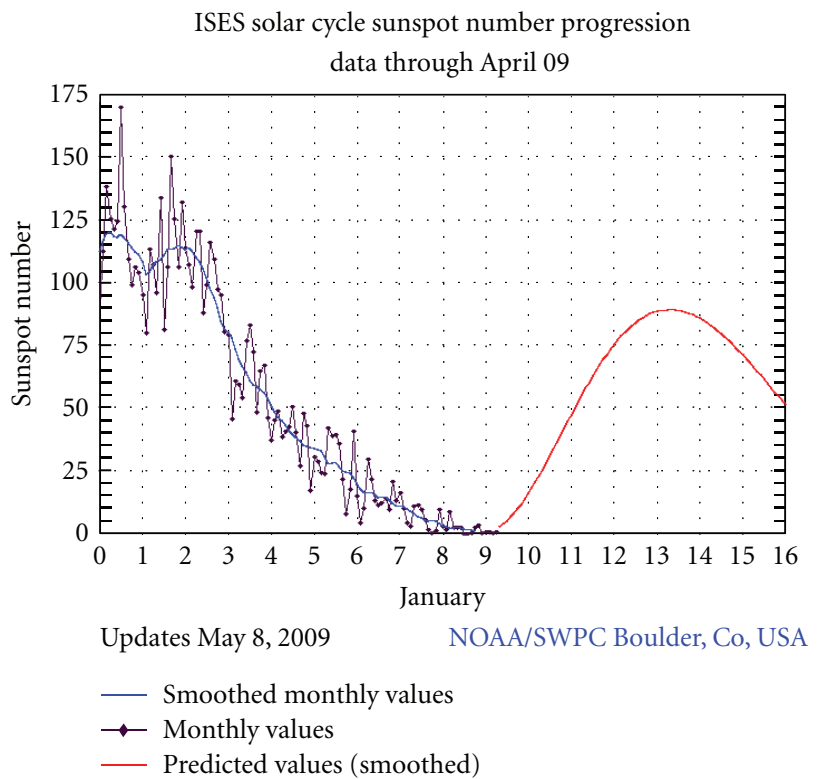

Figure 9: The updated international prediction of SC24 dated April 9, 2009 (http://www.swpc.noaa.gov/SolarCycle/SC24/index.html).

The cosmic ray next minimum is shifted about a year to future in a reference to predicted solar data maximum. The last estimation of the shift between galactic cosmic ray and solar activity variations was 0.6 years [15].

As it is shown by Jan Janssens, the medium 90-140 amp litude SC24 forecasts predominate for today. At present, some researchers conclude that the maximal SC phase has already started. Our modeling allows presuming that researchers have at least a half of a year before monthly mean sunspot maximum will occur and experts have an opportunity to check and update current SC forecasts.

The results obtained from the simulation described above support the relatively high (about $130 \mathrm{Ri}$ ) or moderate magnitude variants of the 24th SC predictions for the monthly mean value case. Forecast capabilities of the modeling method were verified by several retrospective simulations. In the case of the forecast coming true, the entry into a super centurial solar minimum would be shifted in decades to come.

\section{Acknowledgments}

The author would like to thank his closest collaborator Michail Krainev for his significant impact on cosmic ray variation modeling and to express gratitude to heads of the experiments who placed the data in the Internet. Especial acknowledgment has to be expressed to the anonymous reviewer for his valuable comments and recommendations.

\section{References}

[1] M. Dikpati, G. de Toma, and P. A. Gilman, "Predicting the strength of solar cycle 24 using a flux-transport dynamo-based tool," Geophysical Research Letters, vol. 33, no. 5, Article ID L05102, 2006.

[2] M. Dikpati, G. De Toma, and P. A. Gilman, "Polar flux, crossequatorial flux, and dynamo-generated tachocline toroidal flux as predictors of solar cycles," Astrophysical Journal Letters, vol. 675, no. 1, pp. 920-930, 2008.

[3] M. Dikpati, P. A. Gilman, and G. De Toma, "The waldmeier effect: an artifact of the definition of wolf sunspot number?" Astrophysical Journal Letters, vol. 673, no. 1, pp. L99-L101, 2008.

[4] M. Dikpati, "Predicting solar "climate" by assimilating magnetic data into a flux-transport dynamo," Astronomische Nachrichten, vol. 328, no. 10, pp. 1092-1095, 2007.

[5] M. Dikpati and P. A. Gilman, "Simulating and predicting solar cycles using a flux-transport dynamo," Astrophysical Journal, vol. 649, no. 1 I, pp. 498-514, 2006.

[6] L. Svalgaard, E. W. Cliver, and Y. Kamide, "Sunspot cycle 24: smallest cycle in 100 years?" Geophysical Research Letters, vol. 32, no. 1, pp. 1-4, 2005.

[7] R. Brajša, H. Wöhl, A. Hanslmeier et al., "On solar cycle predictions and reconstructions," Astronomy and Astrophysics, vol. 496, no. 3, pp. 855-861, 2009.

[8] K. Petrovay, "Solar cycle prediction," Living Reviews in Solar Physics, vol. 7, pp. 1-59, 2010.

[9] V.I. Kaftan, "Kinematic modeling of the solar activity. 24th solar cycle prediction," in Proceedings of the Experimental and Theoretical Research of the Principles of Solar-Geophysical Activity Predicting, pp. 145-150, Troitsk, Moscow, Russia, 2006, (Russian).

[10] V. I. Kaftan, "Analysis of periodicities of cosmogeophysical processes and Caspian Sea level changes," in Book of Abstracts: Caspian Region: Economics, Ecology, Mineral Resources. International Conference "Caspiy-95”, p. 14, Moscow, Russia, 1995, (Russian).

[11] V. I. Kaftan, Temporal Analysis of Spatial Data: Kinematic Models, Ph.D. thesis, Moscow State University of Transportation, Moscow, Russia, 2003, (Russian).

[12] A. Worthing and J. Geffner, Treatment of Experimental Data, Wiley, New York, NY, USA, 1944. 
[13] G. V. Demianov, V. I. Kaftan, and V. I. Zubinsky, "Participation of the Central Research Institute of Geodesy, Aerial Surveying and Cartography in the third Baltic Sea Level GPS campaign," in Final results of the Baltic Sea Level 1997 GPS campaign, Research Works of the SSC 8.1 of the International Association of Geodesy, M. Poutanen and J. Kakkuri, Eds., pp. 127-132, Finnish Geodetic Institute, Kirkkonummi, Finland, 1999.

[14] V. I. Kaftan, "Kinematic modeling of the main solar cycle," in Multi-Wavelength Investigations of Solar Activity, A. V. Stepanov, E. E. Benevolenskaya, and A. G. Kosovichev, Eds., pp. 111-112, Cambridge University Press, Cambridge, UK, 2004.

[15] V. I. Kaftan and M. B. Krainev, "Estimation of the effect of solar activity on the intensity of galactic cosmic rays," Geomagnetism and Aeronomy, vol. 47, no. 2, pp. 137-148, 2007.

[16] B. Komitov, P. Duchlev, K. Stoychev, M. Dechev, and K. Koleva, "Determination of the sunspot minimum epoch between the cycles No 23 and 24 and prediction of the cycle No 24 magnitude on the base of the 'Waldmeier's Rule," Bulgarian Astronomical Journal, vol. 16, pp. 44-49, 2011.

[17] K. Tapping and J. Valdés, "Did the sun change its behaviour during the decline of cycle 23 and into cycle 24?" Solar Physics, vol. 272, no. 2, pp. 337-350, 2011.

[18] K. L. Harvey, "What is solar cycle minimum?" Journal of Geophysical Research A, vol. 104, no. 9, Article ID 1999JA900211, pp. 19759-19764, 1999. 

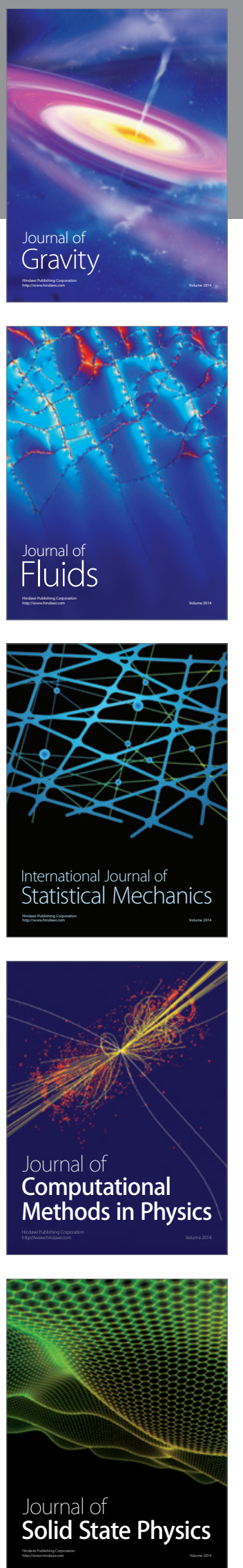

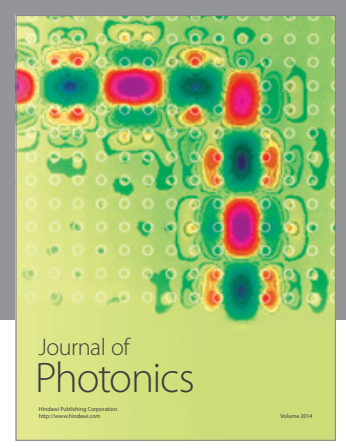

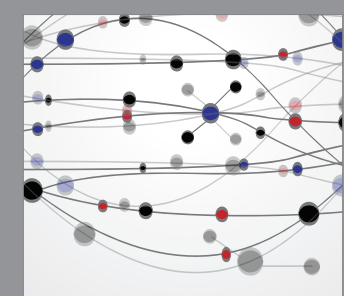

The Scientific World Journal
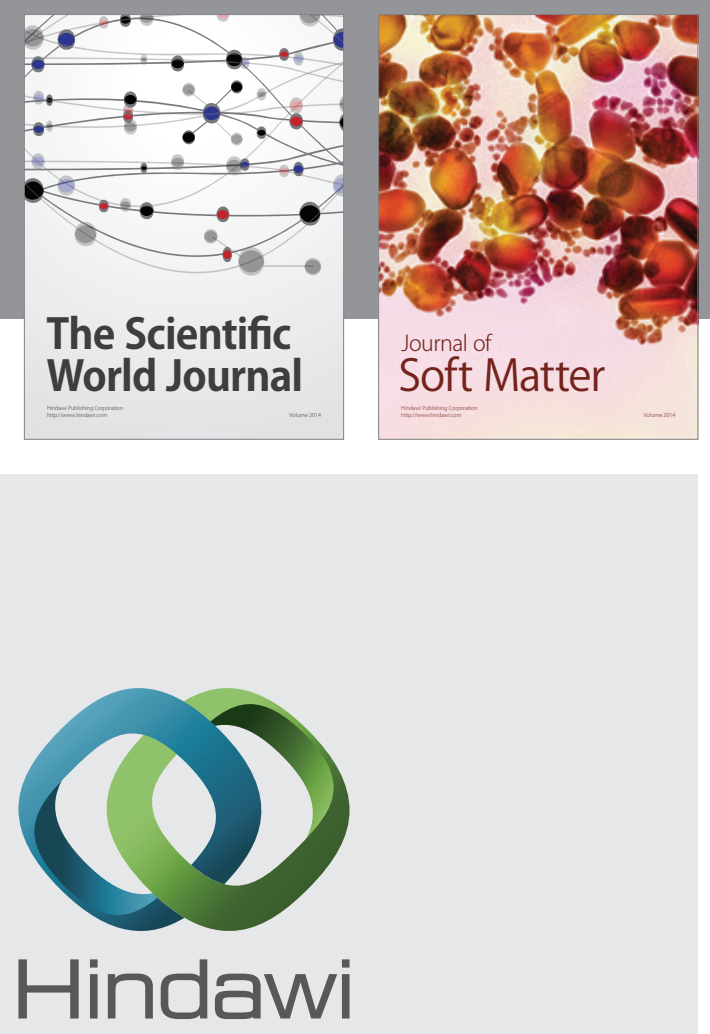

Submit your manuscripts at

http://www.hindawi.com
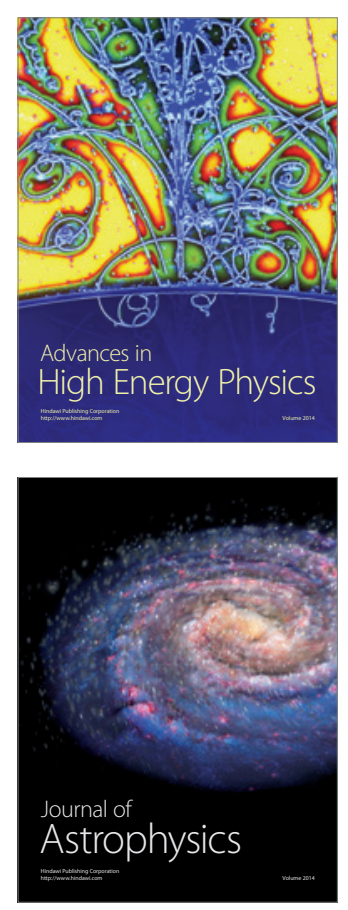
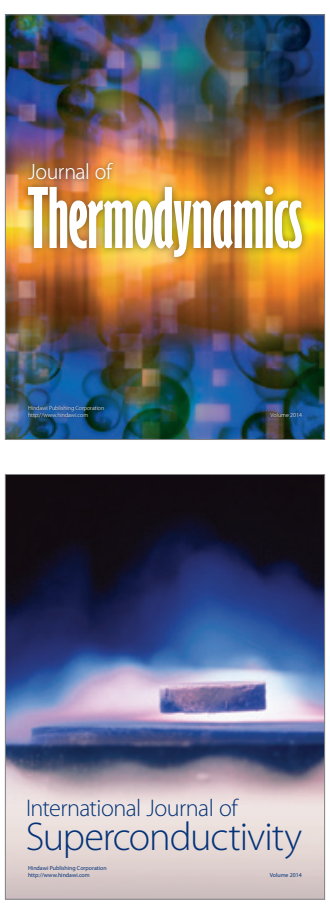
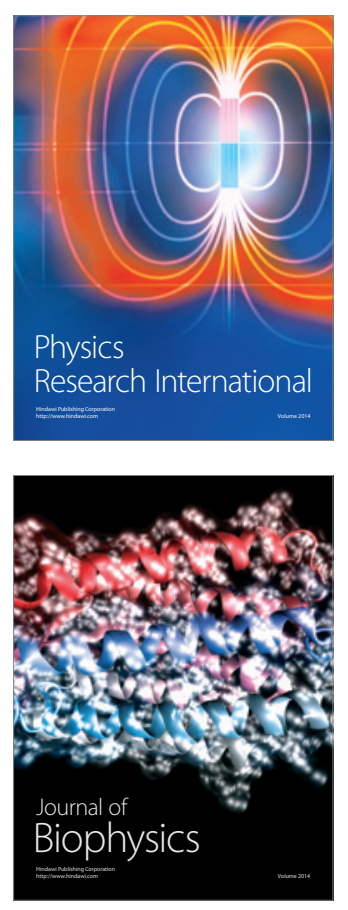
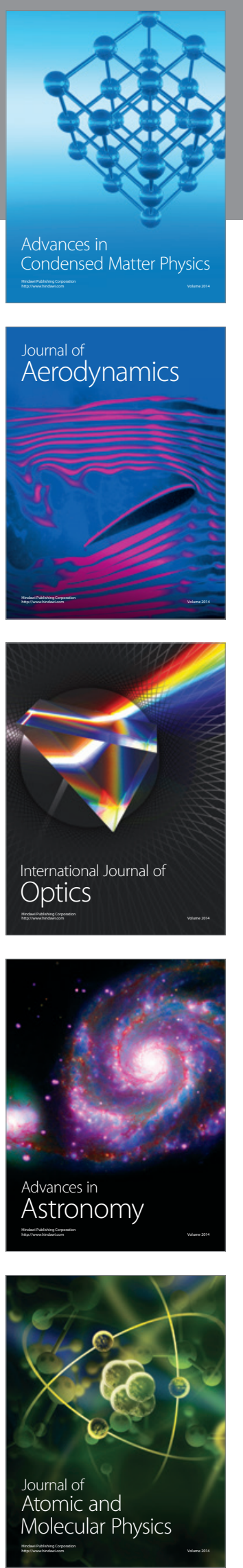